\title{
Clinical features and surgical outcomes of type A intramural hematoma
}

\author{
Yi Shi", Yaojun Dun", Hongwei Guo, Yanxiang Liu, Bowen Zhang, Xiangyang Qian, Cuntao Yu, Xiaogang Sun \\ Department of Cardiovascular Surgery, Fuwai Hospital, National Center for Cardiovascular Diseases, Chinese Academy of Medical Sciences and \\ Peking Union Medical College, Beijing, China \\ Contributions: (I) Conception and design: Y Shi, Y Dun, X Sun; (II) Administrative support: X Sun; (III) Provision of study materials or patients: \\ Y Shi, Y Dun, H Guo; (IV) Collection and assembly of data: Y Dun, Y Liu, B Zhang; (V) Data analysis and interpretation: Y Shi, Y Dun; (VI) \\ Manuscript writing: Y Shi, Y Dun, X Sun; (VII) Final approval of manuscript: All authors. \\ "These authors contributed equally to this work. \\ Correspondence to: Xiaogang Sun. 167\# Beilishi Road, Xicheng District, Beijing, China. Email: xiaogangsunl@163.com.
}

Background: Data on the clinical features and surgical outcomes of type A intramural hematoma (IMH) in Chinese patients are very limited. We aimed to present the surgical experiences on type A IMH in our center, and report early and late outcomes.

Methods: From February 2012 to April 2018, 106 consecutive patients underwent open surgery for type A IMH at our hospital. We adopted emergent operation for patients with cardiac tamponade or other severe complications, and recommended initial medical treatment followed by elective surgery for stable patients. The composite endpoints included operative mortality, permanent nerve damage (stroke, paraplegia), and new-onset renal failure necessitating hemodialysis. Risk factors for operative mortality and the composite endpoints were identified using univariable and multivariable logistic regression model analysis. The survival and freedom from aortic events were analyzed using a Kaplan-Meier surviving curve and a log-rank test.

Results: Except 1 patient receiving emergent surgery (within 24 hours from onset) because of cardiac tamponade and cerebral malperfusion, all patients received initial medical treatment and delayed surgery. Two patient developed pericardial tamponade while waiting for surgery, and then received emergent surgery. Preoperative conversion to aortic dissection (AD) was noted in no patient. The operative techniques included ascending aorta replacement in 9 patients, hemiarch replacement in 18 patients, total arch replacement (TAR) with frozen elephant trunk (FET) in 45 patient and hybrid aortic arch repair in 34 patients. The mean cardiopulmonary bypass (CPB) time and cross-clamp time were $138.7 \pm 41.6$ and $79.3 \pm 27.8 \mathrm{~min}$, respectively. The operative mortality was $1.9 \%$ (2/106). And the composite endpoints occurred in 7 patients. Multivariable logistic regression analysis showed CPB time $\geq 200 \mathrm{~min}$ and chronic kidney disease were risk factors for the composite endpoints. The follow-up data were available in 97 survivors, with the mean followup time of $30.8 \pm 16.2$ months. Three patients died and 5 patients developed aortic events during the followup. The overall survival at 1-, 3- and 5-year were 97.0\%, 95.3\%, and 79.4\%, respectively. And freedom from aortic events at 1-, 3- and 5-year were $97.7 \%, 95.3 \%$ and $89.4 \%$, respectively.

Conclusions: Our strategy had got low mortality and excellent mid-term survival in patients with type A IMH. Therefore, our strategy was suitable for the surgical repair of type A IMH in Chinese population.

Keywords: Intramural hematoma (IMH); aorta; surgical outcomes

Submitted May 29, 2020. Accepted for publication Jul 08, 2020.

doi: $10.21037 /$ jtd-20-748

View this article at: http://dx.doi.org/10.21037/jtd-20-748 


\section{Introduction}

Aortic intramural hematoma (IMH) is a clinical entity characterized by hemorrhage within the aortic wall in the absence of intimal tear and false lumen. This entity account for $10-25 \%$ of acute aortic syndromes (1). IMH results from spontaneous rupture of the vasa vasorum without intimal disruption (2). Less commonly, an intimal disruption of an atherosclerotic plaque could also cause IMH (3). It is generally accepted that the management of type B IMH is primarily medical treatment (4). However, controversy still exists regarding the optimal treatment of type A IMH. Considering poor prognosis of medical treatment (5), some Western investigators advocated early surgery $(1,6)$. However, other studies from Japan and South Korea have demonstrated excellent early outcomes with initial medical treatment and timely surgery (7-9). It is unknown whether the latter strategy is applicable to Chinese population. And data on the clinical features and surgical outcomes of type A IMH in Chinese patients are very limited. Therefore, the purpose of this study was to present our experiences with surgical repair of type A IMH and report early and late outcomes. We present the following article in accordance with the STROBE reporting checklist (available at http:// dx.doi.org/10.21037/jtd-20-748).

\section{Methods}

\section{Patients selection}

The study was conducted in accordance with the Declaration of Helsinki (as revised in 2013). The study protocol was approved by the Institutional Ethics Committee of Fuwai Hospital. And a waiver of informed consent was granted due to the design of the study.
Between February 2012 and April 2018, 106 patients with type A IMH were admitted to Fuwai hospital and received surgery. The diagnosis was established by computed tomography (CT). IMH was defined as a circular or crescentic thickening of $>5 \mathrm{~mm}$ of the aortic wall, without evidence of blood flow on imaging examination (10). Patients who showed concurrent ulcer-like projection (ULP) or penetrating atherosclerotic ulcer (PAU) into the hematoma space on the CT, and patients with preoperative conversion to typical aortic dissection (AD) were also included in this study. On admission, 56 patients presented with concurrent ascending/arch ULP, and 1 patient presented with concurrent ascending/arch PAU. The preoperative CT scans of patients with IMH alone, concurrent ULP and concurrent PAU are shown in Figure 1.

\section{Data collection and definitions}

All clinical data were collected from electronical medical record system. Acute IMH referred to admission within the first 2 weeks, whereas subacute IMH referred to admission between 2 weeks and 3 months after the initial event. And patients were divided into acute IMH group and subacute IMH group. Respiratory failure was defined as prolonged intubation (more than 48 hours), reintubation and requirement for tracheostomy. Renal failure was defined as percentage increase in postoperative serum creatinine of beyond $100 \%$, or renal failure necessitating hemodialysis. Operative mortality was defined as death within 30 days of surgery or before final discharge from hospitalization. The composite endpoints included operative mortality, permanent nerve damage (stroke, paraplegia), and newonset renal failure necessitating hemodialysis. Aortic events were defined as follows: new $\mathrm{IMH}$ or $\mathrm{AD}$, aortic rupture,
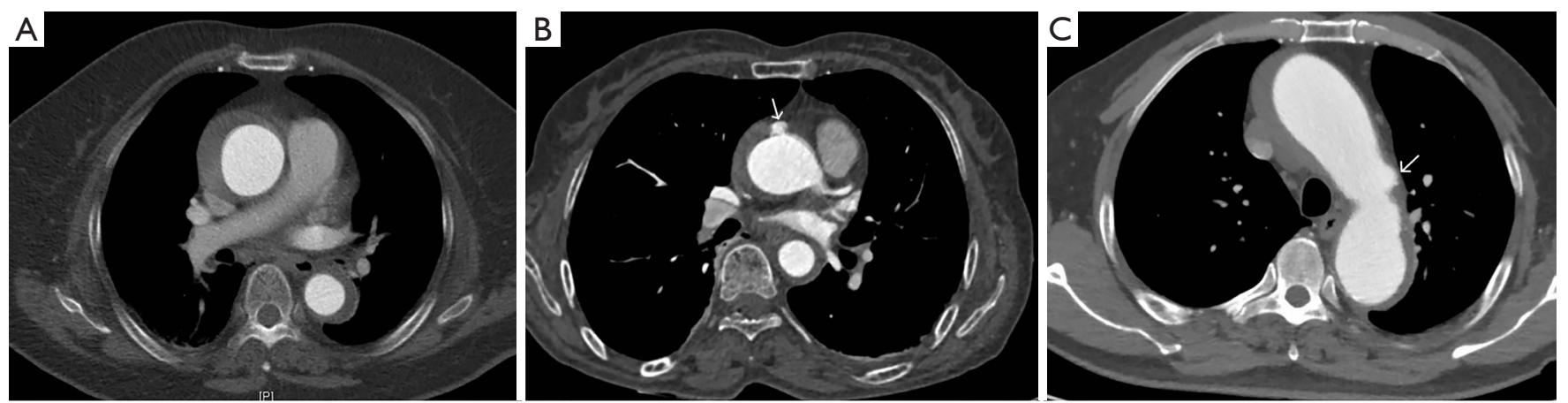

Figure 1 Preoperative computed tomography scans. (A) IMH alone; (B) IMH with concurrent ulcer-like projection (arrowhead); (C) IMH with concurrent penetrating atherosclerotic ulcer (arrowhead). IMH, intramural hematoma. 

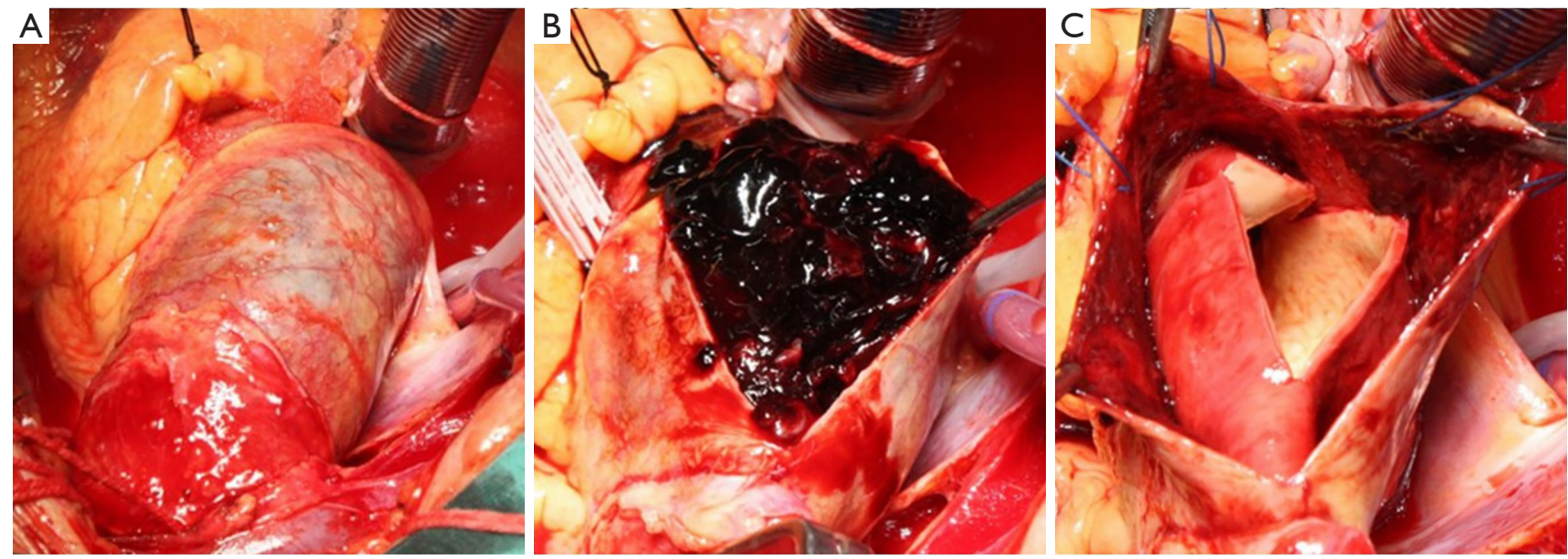

Figure 2 Intraoperative findings of type A IMH. (A) The outside view; (B) the view after incision of the adventitia; (C) the view after incision of the aortic wall. IMH, intramural hematoma.

aortic related surgical treatment, death of aortic cause and unexplained death.

\section{Operative strategy}

Our indication of surgery for IMH included cardiac tamponade, an ascending aorta of $50 \mathrm{~mm}$ or greater, and thickness of a hematoma greater than $11 \mathrm{~mm}$, concurrent PAU/ULP involving ascending aorta/arch, concurrent type B AD. We adopted emergent operation for patients with cardiac tamponade or other severe complications (aortic rupture or periaortic hematoma, major organ malperfusion (brain, coronary)). And we recommended initial medical treatment (pain control, blood pressure and heart rate control) followed by elective surgery for stable patients. For patients with serious comorbidities including heart failure, hepatic insufficiency, renal failure and respiratory failure, the operative risk was very high, and surgery should be considered until they recovered to relatively normal organ function. CT was repeated for patients with new symptoms while waiting for surgery. Emergent surgery was performed if previous described emergent operative indications were met or the hematoma was converted to classical AD. If the ascending aorta enlarged, the hematoma thickened, new ULP emerged or ULP progressed (width or depth increased) on CT, we also recommended timely surgery (within 72 hours). We determined different operative procedures according to the involvement of hematoma and the location of ULP or PAU. For patients with hematoma involving ascending aorta or proximal arch, we adopted ascending aorta or hemiarch replacement.

Our indication of total arch replacement (TAR) was aortic arch dilatation $(\geq 50 \mathrm{~mm})$, a PAU or ULP in the distal aortic arch, and concurrent type B AD. An additional frozen elephant trunk (FET) technique was always performed in combination with TAR, especially for patients with distal arch or descending aortic lesions (dissection, PAU, ULP). This procedure could move the distal anastomosis plane from Zone 3 to Zone 2, which could facilitate the distal anastomosis and decrease the risk of anastomotic bleeding. The FET graft used at our institution (Cronus, MicroPort Endovascular, Shanghai Co., Ltd, Shanghai, China) had an extra centimeter of attached Dacron graft proximally, which also made the distal arch reconstruction more stable and convenient. These indications for TAR also applied to hybrid aortic arch repair. Patients with age more than 65 years old, or complicated arch pathology or malperfusion syndrome carried high risks for TAR. In these patients with good economic state, we recommended hybrid aortic arch repair. These criteria were relative factors in decisionmaking process, not absolute indications. The final decision depended on the operating surgeon.

All operations were performed via median sternotomy (Figure 2). The vein cannula was placed in the right atria, and arterial cannulation was placed in different sites (the right axillary artery, the innominate artery, and the femoral artery), based on patient's characteristics. And the right axillary artery or the innominate artery was selected for selective antegrade cerebral perfusion (SCP). Nasopharyngeal temperature was lowered to $28{ }^{\circ} \mathrm{C}$ for 
moderate hypothermic cardiopulmonary bypass $(\mathrm{CPB})$, or $20-25{ }^{\circ} \mathrm{C}$ for hypothermia circulatory arrest (HCA) with SCP. HCA was required in all patients receiving TAR, and some patients receiving hemiarch replacement. For the other patients who received hemiarch replacement without HCA, the distal anastomosis was performed with the clamping of distal arch and the innominate artery, and the distal perfusion was achieved through the femoral artery cannulation.

The detailed operative procedure of hybrid aortic arch repair has been previously described by our colleagues (11). This operation was performed under moderate hypothermic $\mathrm{CPB}$. After reconstruction of Zone 0 and Zone 1 with a four-branched vascular prosthesis (Terumo, Vascutek Limited, Renfrewshire, UK), arch debranching of the 3 supra-aortic branches was performed. Then the stent graft could be retrogradely deployed with the proximal landing zone of Dacron Zone 0. The stent graft was oversized by $10-15 \%$. Five kinds of commercial stents were applied at our institution, including Zenith (Cook Medical Inc, Bloomington, Ind), Relay (Bolton Medical, Sunrise, Fla), Talent and Valiant (Medtronic Inc, Santa Rosa, Calif), Hercules (MicroPort Medical Co, Ltd, Shanghai, China), and Ankura (Lifetech, Shenzhen, China).

For patients who received concomitant Bentall procedure in the surgical repair of IMH, we adopted the inclusion technique with a perigraft-to-right atria shunt. During the operation, we did not remove the aneurysmal wall. After composite graft replacement of aortic root within the aneurysmal sac, we used the residual aortic wall to wrap around the graft. Then we made an incision at the roof of the right atrial appendage and performed a direct perigraftto-right atria anastomosis, to create a perigraft-to-right atria shunt.

\section{Follow-up and statistical analysis}

The follow-up data were obtained by direct telephone contact or at the outpatient clinic. Morbidity, aortic events and mortality during the follow-up were recorded. CT was performed at regular interval after surgery.

Continuous variables were described as mean \pm standard deviation (SD) or median (range), and categorical variables as number (\%). The Student $t$-test or Mann-Whitney $\mathrm{U}$ test was used for the comparison of the continuous variables as appropriate. Between-group comparison for categorical variables was performed by chi-square test or Fisher exact test. Risk factors for operative mortality and the composite endpoints were identified using univariable and multivariable logistic regression model analysis. Midterm survival and freedom from aortic events were analyzed using a Kaplan-Meier surviving curve and a log-rank test. All statistical analyses were performed with SPSS software, version 21 (SPSS Inc., Chicago, IL). A P value $<0.05$ was regarded as statistically significant.

\section{Results}

\section{Baseline characteristics}

The mean age was $57.9 \pm 10.6$ years, and 58 patients $(54.7 \%)$ were female. The average body mass index was higher in acute IMH group than that in subacute IMH group $(\mathrm{P}=0.041)$. The demographic characteristics is shown in Table 1.

The most common symptoms included chest pain, back pain, abdominal pain, loss of consciousness. Two patients had received pericardial drainage for massive pericardial effusion before admission (in the local hospital). Five patients presented with organ malperfusion and 5 patients with hypotension. After admission, 1 patient required tracheal intubation for respiratory failure, and another patient required hemodialysis for renal failure. The average diameter of ascending aorta and thickness of hematoma were $50.6 \pm 6.5 \mathrm{~mm}, 13.8 \pm 4.8 \mathrm{~mm}$, respectively. The proportion of patients with distal involvement of hematoma at descending aorta and abdominal aorta was higher in acute IMH group than that in subacute IMH group $(74.7 \%$ vs. $51.9 \%, \mathrm{P}=0.027 ; 49.4 \%$ vs. $18.5 \%, \mathrm{P}=0.005$, respectively). The preoperative characteristics is illustrated in Table 2.

\section{Operative data}

Except 1 patient receiving emergent surgery (within 24 hours from onset) because of cardiac tamponade and cerebral malperfusion (Figure 3), all patients received initial medical treatment and delayed surgery. Two patient developed pericardial tamponade while waiting for surgery, and then received emergent surgery. CT was repeated in 13 patients for new symptoms during the waiting period. Five patients presented with new ULP, and 2 patients presented with thickened hematoma on CT. They received timely operation except 1 patient with new ULP and pericardial tamponade received emergent surgery (Figure 4), while the remaining 6 patients with no change in IMH received elective surgery. And no patient had 
Table 1 Demographic characteristics

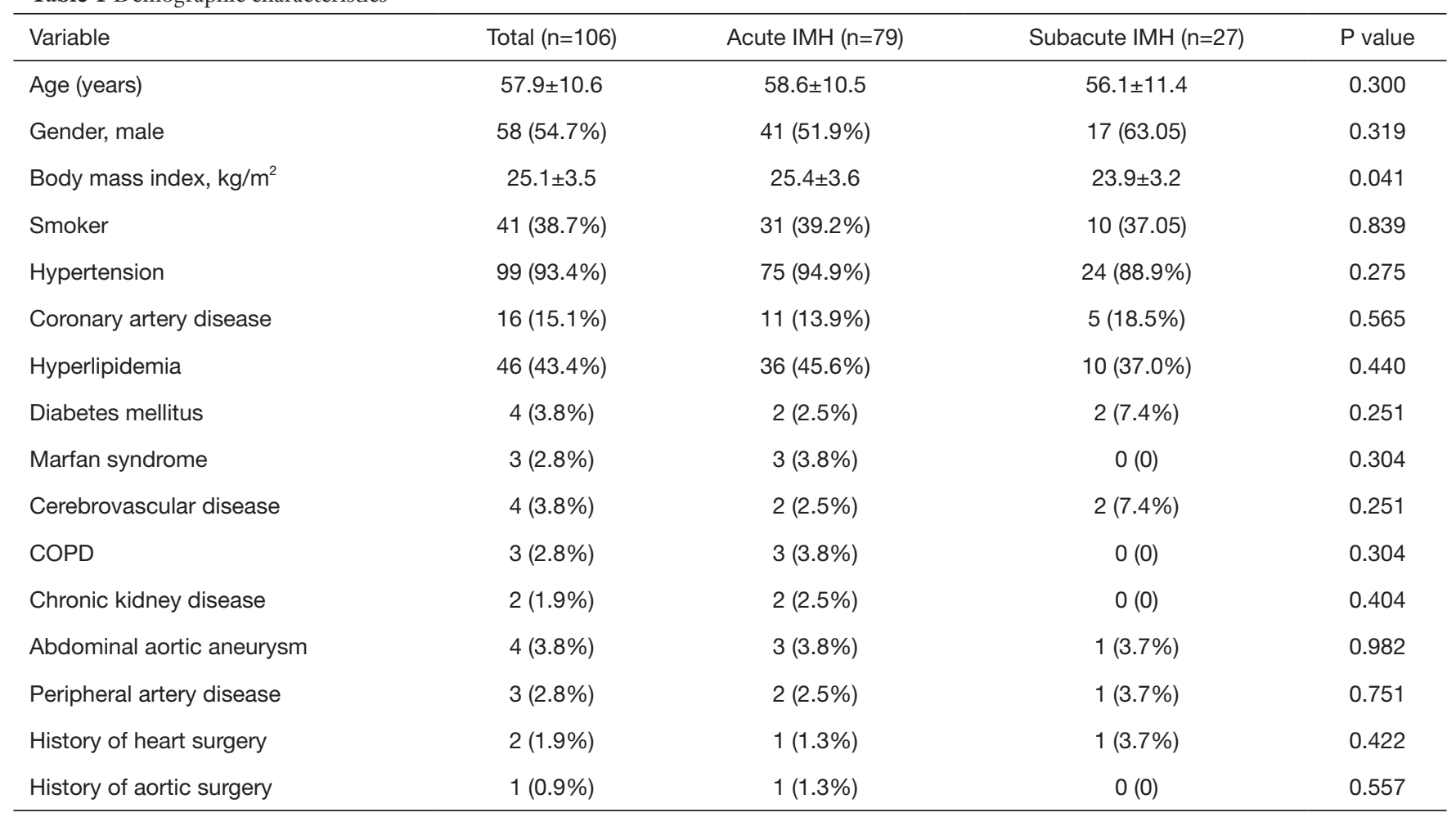

$\mathrm{IMH}$, intramural hematoma; COPD, chronic obstructive pulmonary disease.

preoperative conversion to AD. The median interval between admission and operation was 114 hours (range, 7-923 hours). And 93 patients (87.7\%) received delayed operation (beyond 72 hours from disease onset).

During the surgery, aortic intima tear was noted in 81 patients $(76.4 \%)$, including 28 patients $(57.1 \%, 28 / 49)$ with IMH alone, 52 patients $(92.9 \%, 52 / 56)$ with concurrent ULP, 1 patient $(100 \%, 1 / 1)$ with concurrent PAU. The operative procedures included ascending aorta replacement in 9 patients, hemiarch replacement in 18 patients, TAR with FET in 45 patient and hybrid aortic arch repair in 34 patients. Twenty three patients underwent additional procedures, including Bentall procedure, David procedure, coronary artery bypass grafting (CABG), aortic valve repair/ replacement, endovascular abdominal aneurysm repair. The mean CPB time and cross-clamp time were $138.7 \pm 41.6$ and $79.3 \pm 27.8 \mathrm{~min}$, respectively. HCA was required in 55 patients (51.9\%), including 45 patients receiving TAR with FET, and 10 patients receiving hemiarch replacement. And the mean HCA duration was $18.5 \pm 6.1 \mathrm{~min}$. The operative details are displayed in Table 3.

\section{Early postoperative outcomes}

The operative mortality was $1.9 \%(2 / 106)$, and both 2 patients died of multiple organ failure. The composite endpoints occurred in 7 patients, all in acute IMH group. The average ventilation duration was longer in acute IMH group than that in subacute IMH group $(\mathrm{P}=0.004)$. The incidence of postoperative renal failure was higher in acute IMH group than that in subacute IMH group $(13.9 \%$ vs. $0, \mathrm{P}=0.041)$. The postoperative results are also showed in Table 3.

No variable was identified to be the risk factor for operative mortality on regression analysis. And univariable logistic regression analysis showed $\mathrm{CPB} \geq 200 \mathrm{~min}$ was the risk factor for the composite endpoints [odds ratio (OR) $11.63,95 \%$ confidence interval (CI): 2.10-64.24, $\mathrm{P}=0.005$ ], while multivariable logistic regression analysis showed $\mathrm{CPB}$ time $\geq 200 \mathrm{~min}$ and chronic kidney disease were risk factors for the composite endpoints (OR 26.78, 95\% CI: 1.88381.62, $\mathrm{P}=0.015$ and OR 44.40, 95\% CI: $1.18-1677.18$, $\mathrm{P}=0.041$, repectively). 
Table 2 Preoperative patient characteristics

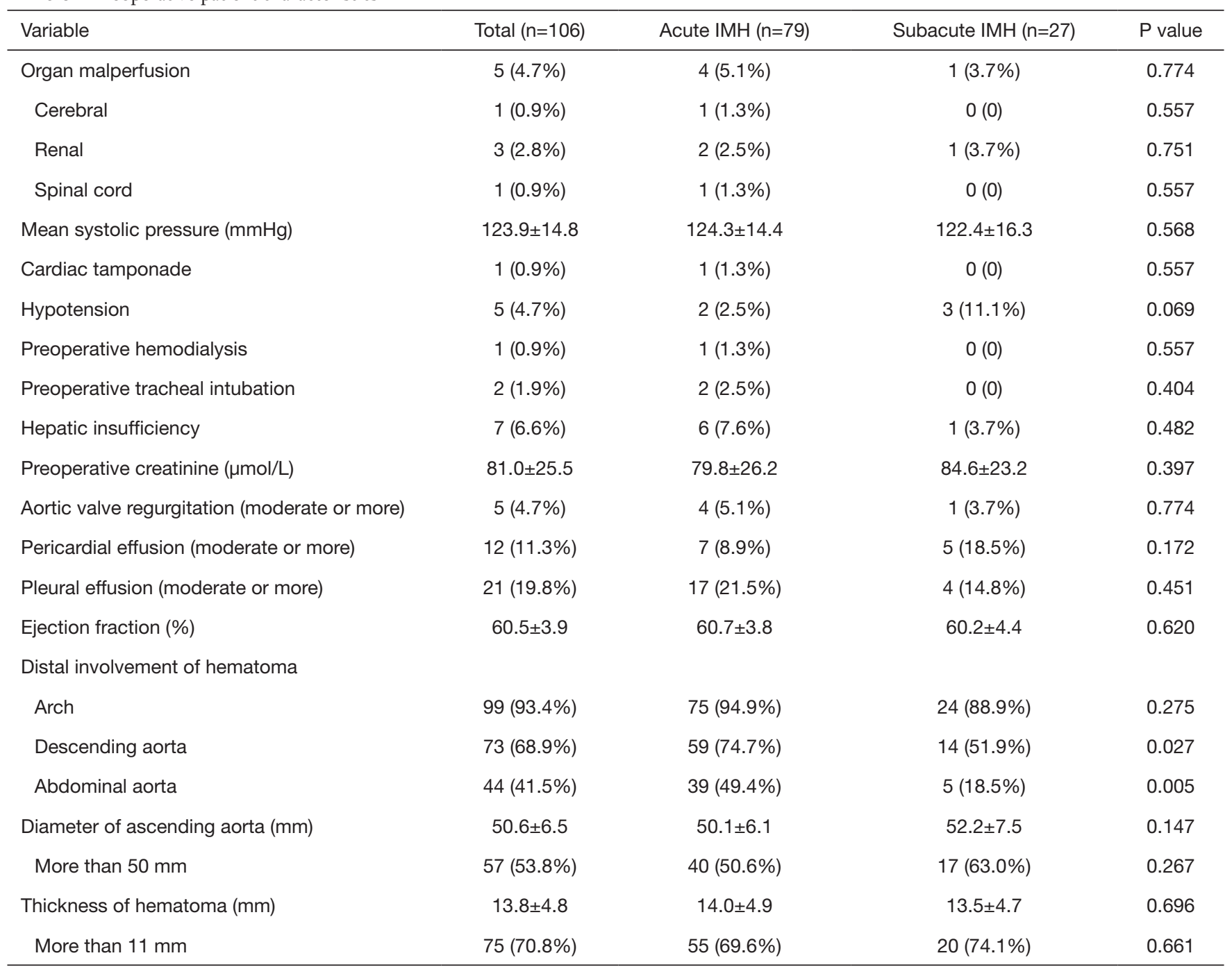

$\mathrm{IMH}$, intramural hematoma.

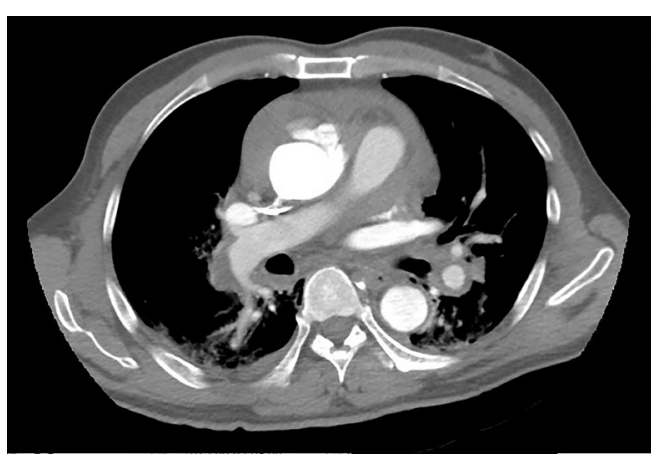

Figure 3 The patient was diagnosed with type A IMH and ulcer-like projection of root on computed tomography scan. $\mathrm{He}$ received emergent surgery for pericardial tamponade and cerebral malperfusion. IMH, intramural hematoma.

\section{Mid-term outcomes}

The follow-up data were available in 97 survivors. The mean follow-up time was $30.8 \pm 16.2$ months (1-73 months). Three patients died and 5 patients developed aortic events during the follow-up. The causes of death and aortic events are shown in Figure 5. The overall survival at 1 -year, 3 -year and 5 -year were $97.0 \%, 95.3 \%$, and $79.4 \%$, respectively (Figure $6 \mathrm{~A}$ ). And freedom from aortic events at 1-year, 3-year and 5-year were $97.7 \%, 95.3 \%$ and $89.4 \%$, respectively (Figure 6B). There were no significant differences in overall survival and freedom from aortic events between acute IMH group and subacute IMH group $(\mathrm{P}=0.095$ and $\mathrm{P}=0.742$, respectively) 

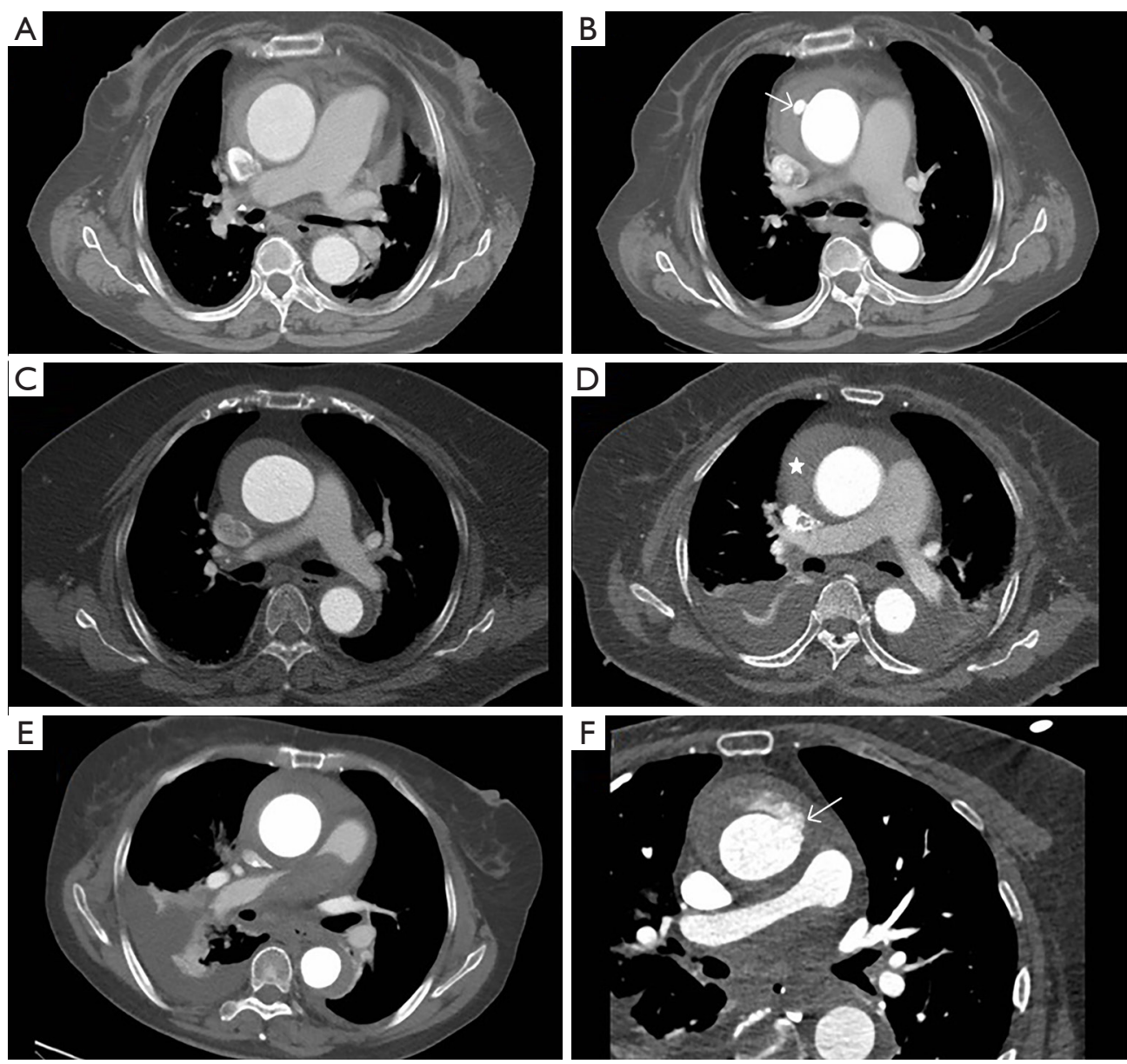

Figure 4 Preoperative computed tomography (CT) scans of three patients who presented with disease progression on second CT. (A) First CT of case 1; (B) second CT of case 1 shows new ulcer-like projection (arrowhead); (C) first CT of case 2; (D) second CT of case 2 shows thickened hematoma (asterisk); (E) first CT of case 3; (F) second CT of case 3 shows a large ulcer-like projection (arrowhead); the patient also presented with pericardial tamponade and received emergent surgery.

(Figure $7 A, B)$.

\section{Discussion}

IMH was first introduced by Krukenberg in 1920 as "AD without intimal tear", and was considered a distinct pathologic entity among aortic diseases (12). The prevalence of IMH is reported to be $10-25 \%$ among patients with acute aortic syndromes (1). However, controversy still exists regarding the optimal treatment of type A IMH. Interestingly, according to several investigators, type A IMH may be significantly more common in Japan/Korea than in Western countries $(13,14)$. And most studies from Japan/Korea favored early medical treatment in the absence of serious complications, while the investigators from the Western countries recommended early surgical treatment (14).

In some Asian studies, single medical treatment for patients with type A IMH meeting specific criteria could get acceptable early and late outcomes $(15,16)$. Some investigators also reported that medical treatment could provide complete absorption of hematoma and favorable clinical outcomes $(17,18)$. However, patients receiving single medical treatment carried risks of IMH progression, aortic dilation, conversion to $\mathrm{AD}$, aortic rupture or sudden death during the follow-up (16,18-20). Therefore, other investigators recommended initial medical treatment followed by timely surgery for stable patients, and emergent surgical repair for unstable patients with severe complications on admission $(7,8,21)$. All the above 
Table 3 The operative and postoperative results

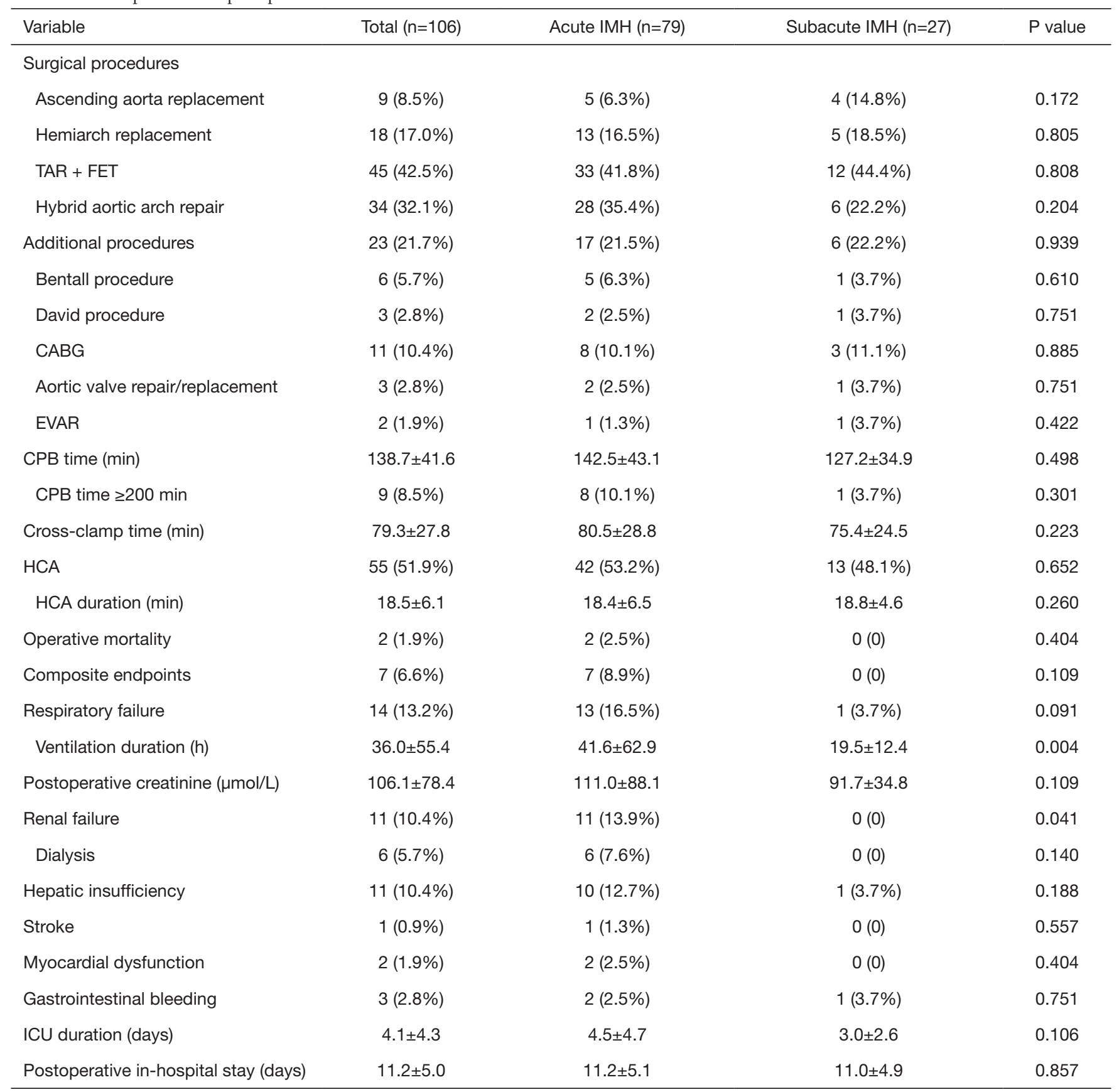

$\mathrm{IMH}$, intramural hematoma; TAR, total arch replacement; FET, frozen elephant trunk; CABG, coronary artery bypass grafting; EVAR, endovascular aneurysm repair; CPB, cardiopulmonary bypass; HCA, hypothermic cardiac arrest; ICU, intensive care unit.

studies were from Japan/Korea, and they advocated initial medical treatment for stable patients because of lower overall mortality $(13,14)$. And Estrera and colleagues (22) from United States had identified that $33 \%$ of cases converted to typical AD by the time of operation and the risk of conversion was greatest at 8 days from the onset of symptoms. Considering low risk for early conversion, they also adopted a strategy to perform delayed operation (within 3 days of presentation) for patients with type A IMH (6).

However, the mortality was 6 times higher for patients with 
A
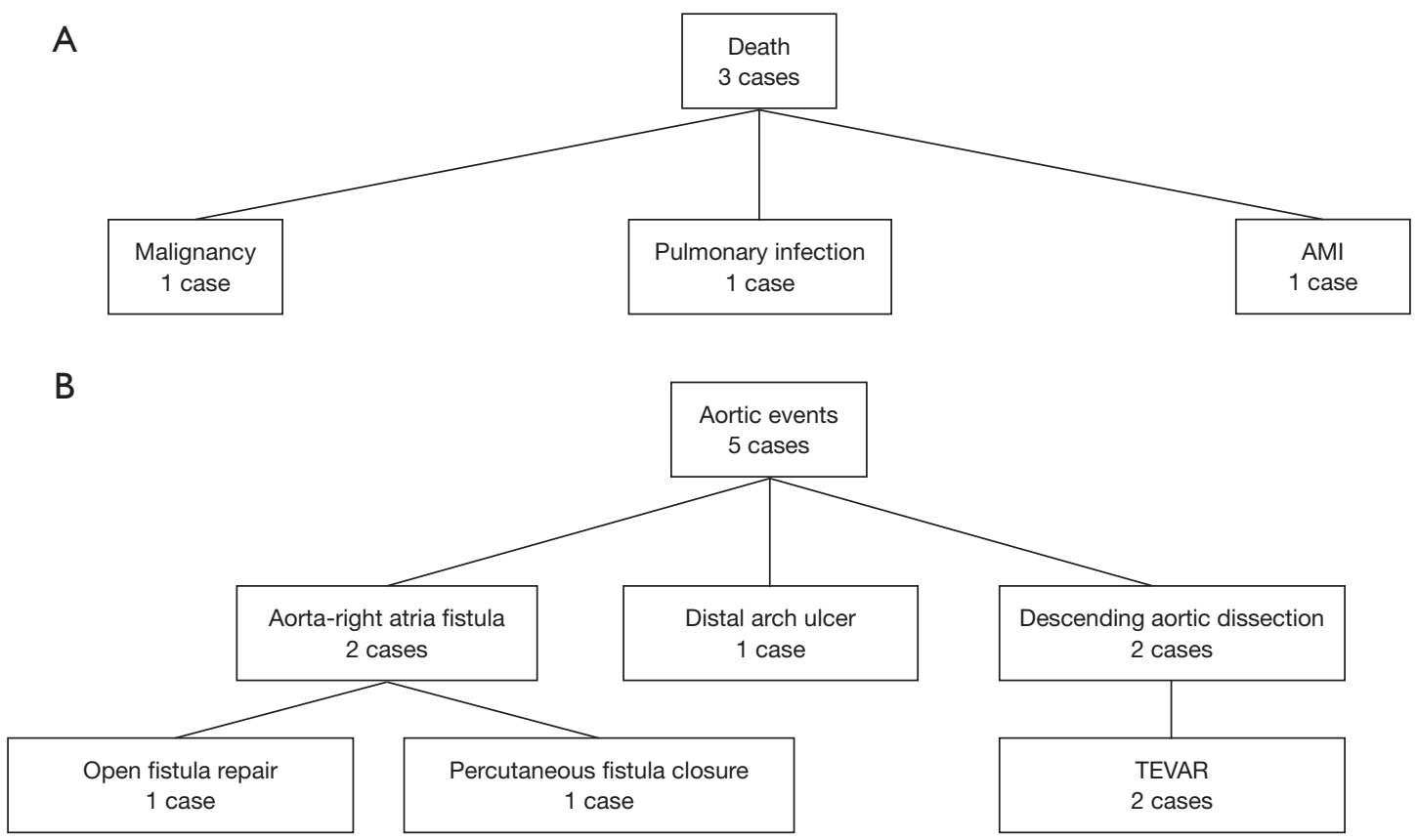

Figure 5 The causes of deaths and aortic events during the follow-up. (A) the causes of deaths; (B) the causes and management of aortic events. AMI, acute myocardial infarction; TEVAR, thoracic endovascular aortic repair.

A

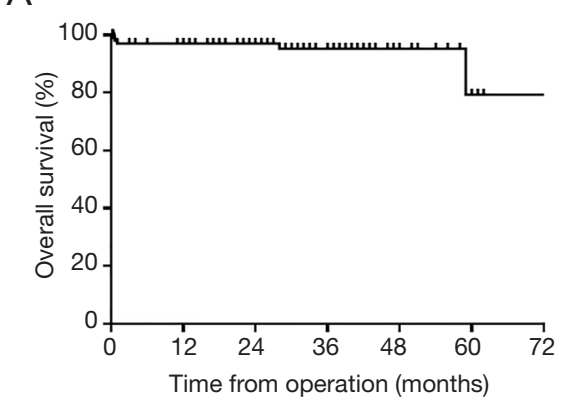

Patients at risk

$\begin{array}{lllllll}106 & 86 & 66 & 42 & 15 & 5 & 3\end{array}$
B

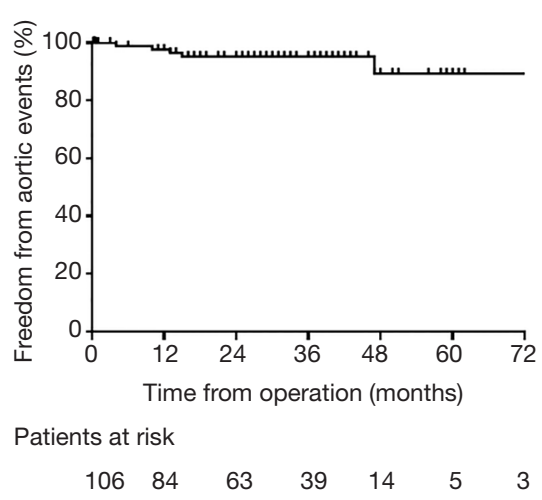

Figure 6 Kaplan-Meier surviving curves. (A) Overall survival; (B) freedom from aortic events.

type A IMH who were treated medically in Western countries compared with those in Japan/Korea, while the mortality in patients who received early surgery did not differ between the 2 regions (14). Therefore, most Western investigators favored emergency surgery for patients with type A IMH $(23,24)$. And another 2 publications from Japan during the last 5 years also advocated emergency surgical treatment for all patients with type A IMH $(20,25)$. Hata and colleagues (20) reported 171 patients with type A IMH and divided them into 3 groups according to complication status: groups I and
II consisted of 74 and 33 patients undergoing emergency surgery for complicated and uncomplicated type A IMH, with mortality of $5.4 \%$ and 0 , respectively, and group III comprised 66 patients who were treated conservatively, with mortality of $25.8 \%$. Therefore, they advocated emergent operation for all patients with type A IMH, which could provide better outcome than medical treatment (20).

At our institution, we adopted medical treatment alone for patients meeting the following criteria: aortic diameter $<50 \mathrm{~mm}$, IMH thickness $<11 \mathrm{~mm}$, without concurrent 
A

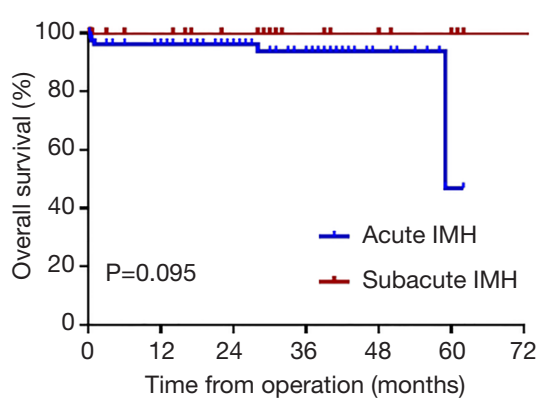

Patients at risk

$\begin{array}{llllllll}\text { Acute } & 79 & 67 & 51 & 33 & 9 & 2 & 1\end{array}$

B

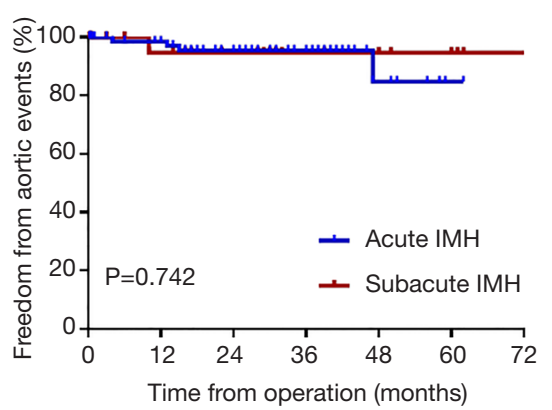

Patients at risk

$\begin{array}{llllllll}\text { Acute } & 79 & 66 & 49 & 31 & 9 & 2 & 1\end{array}$

Figure 7 The comparison of Kaplan-Meier surviving curves between acute IMH group and subacute IMH group. (A) Overall survival; (B) freedom from aortic events. IMH, intramural hematoma.

ULP/PAU, stable hemodynamics. Otherwise, surgery was indicated. And we adopted emergent operation for patients with cardiac tamponade or other severe complications, and recommended initial medical treatment followed by elective surgery for stable patients with type A IMH. The clinical outcomes were favorable both in postoperative course and mid-term follow-up. Our institution was the most famous cardiovascular center in China, and most of our patients with IMH were referred from other remote hospitals. Many high-risk patients who were contraindicative to operation and those who denied operation, and unstable patients who died before admission might not be included in our cohort. And most of patients in this study did not require emergent surgery and should have been in stable condition. That could partly explain operative mortality of only $1.9 \%$, which was much less than mortality from other studies [Estrera et al. 10.9\% (7/64) (6) and Hata et al. 3.7\% (4/107) (20)]. This study was also the largest cohort of type A IMH in Chinese population to date, so we believed that our strategy was reliable and could be widely applied in Chinese population. Estrera and colleagues (6) demonstrated timely surgery within the first 3 days of presentation could decrease the mortality of type A IMH. We just advocated elective surgery for stable patients with type A IMH, with no definite requirement of surgery timing. We hypothesized that a purposeful delay in operation may ensure the inflammatory response to subside and allow tissues to thicken and stabilize, which may contribute to more favorable operative outcomes (26). In the clinical practice of last 2 years, we preferred elective surgery within 5 days of admission.

In acute IMH group, 2 patients died postoperatively while no patient died in subacute IMH group. However, acute IMH was not identified a risk factor for operative mortality and the composite endpoints. Only CPB time $\geq 200 \mathrm{~min}$ and chronic kidney disease were identified to be risk factors for the composite endpoints. We inferred that longer CPB time and preoperative chronic kidney disease could increase incidence of postoperative renal failure. In addition, additional procedures were often associated with longer CPB time. Among 9 patients with CPB time $\geq 200$ min, 5 patients had additional procedures, including CABG in 4 patients, Bentall procedure in 1 patient and David procedure in 1 patient. After univariable and multivariable analysis, the additional procedure or CABG or root procedure was not identified to be a risk factor for the composite endpoints.

During the follow-up, aortic events occurred in 5 patients. Thus, we thought a long-term periodical followup was necessary because the late reoperation rate was not low. Two patients in this group suffered aorta-right atria fistula and they received open fistula repair or percutaneous fistula closure. Both patients received additional Bentall procedure with the graft inclusion technique during the primary operation. The point of this technique was to create the connection between the perigraft and the right atria by direct anastomosis, which could control the surgical bleeding and improve hemostasis of aortic root and coronary anastomosis. This procedure could decrease the risk of postoperative bleeding. The perigraftto-right atria shunt would close spontaneously as the bleeding stopped and thrombus accumulated inside the perigraft compartments. But for patients with persistent anastomotic bleeding (coronary ostia, aortic root) after 
surgery, the perigraft-o-right atria shunt could not close and turn into the persistent aorta-right atria fistula, which was not an uncommon complication during the followup (27). Pseudoaneurysm, coronary artery-to-perigraft fistula, right heart failure because of persistent left-to-right shunt were also the late complications after this procedure. And many patients required reinterventions to treat these complications. Considering the late outcome, more and more surgeons at our institution adopted Bentall procedure with the button technique instead of the inclusion technique in the surgical repair of type A IMH or AD during the last 2 years.

The present study had several limitations. This study was retrospective, observational study, and the sample size was relatively small. And this was a single-center study, and there was selection bias of patients population. We did not explore the optimal timing of surgical intervention for type A IMH in this study. And our results may not apply to the Western population. We only included IMH cases receiving surgical treatment, and most cases received elective surgery. Hence, a larger randomized multi-center study including non-surgical IMH cases and cases receiving early surgery will be necessary to elucidate the clinical picture of type A IMH.

\section{Conclusions}

In conclusion, we adopted emergent operation for unstable patients with type A IMH (cardiac tamponade or other severe complications), and recommended initial medical treatment and elective surgery for stable patients with type A IMH. This strategy showed low mortality and satisfactory mid-term results in our study, and it could be applied in Chinese population.

\section{Acknowledgments}

Funding: This work was supported by Special fund for clinical research of Chinese Medical Association (No. 14010020539).

\section{Footnote}

Reporting Checklist: The authors have completed the STROBE reporting checklist. Available at http://dx.doi. org/10.21037/jtd-20-748

Data Sharing Statement: Available at http://dx.doi. org/10.21037/jtd-20-748

Conflicts of Interest: All authors have completed the ICMJE uniform disclosure form (available at http://dx.doi. org/10.21037/jtd-20-748). The authors have no conflicts of interest to declare.

Etbical Statement: The authors are accountable for all aspects of the work in ensuring that questions related to the accuracy or integrity of any part of the work are appropriately investigated and resolved. The study was conducted in accordance with the Declaration of Helsinki (as revised in 2013). The study protocol was approved by the Institutional Ethics Committee of Fuwai Hospital. And a waiver of informed consent was granted due to the design of the study.

Open Access Statement: This is an Open Access article distributed in accordance with the Creative Commons Attribution-NonCommercial-NoDerivs 4.0 International License (CC BY-NC-ND 4.0), which permits the noncommercial replication and distribution of the article with the strict proviso that no changes or edits are made and the original work is properly cited (including links to both the formal publication through the relevant DOI and the license). See: https://creativecommons.org/licenses/by-nc-nd/4.0/.

\section{References}

1. Erbel R, Aboyans V, Boileau C, et al. 2014 ESC Guidelines on the diagnosis and treatment of aortic diseases:

Document covering acute and chronic aortic diseases of the thoracic and abdominal aorta of the adult. The Task Force for the Diagnosis and Treatment of Aortic Diseases of the European Society of Cardiology (ESC). Eur Heart J 2014;35:2873-926.

2. Gore I. Pathogenesis of dissecting aneurysm of the aorta. AMA Arch Pathol 1952;53:142-53.

3. Stanson AW, Kazmier FJ, Hollier LH, et al. Penetrating atherosclerotic ulcers of the thoracic aorta: natural history and clinicopathologic correlations. Ann Vasc Surg 1986;1:15-23.

4. Moizumi Y, Komatsu T, Motoyoshi N, et al. Clinical features and long-term outcome of type A and type $\mathrm{B}$ intramural hematoma of the aorta. J Thorac Cardiovasc Surg 2004;127:421-7.

5. Tittle SL, Lynch RJ, Cole PE, et al. Midterm follow-up of penetrating ulcer and intramural hematoma of the aorta. J 
Thorac Cardiovasc Surg 2002;123:1051-9.

6. Estrera AL, Sandhu HK, Leake SS, et al. Early and late outcomes of acute type A aortic dissection with intramural hematoma. J Thorac Cardiovasc Surg 2015;149:137-42.

7. Kitai T, Kaji S, Yamamuro A, et al. Clinical outcomes of medical therapy and timely operation in initially diagnosed type a aortic intramural hematoma: a 20-year experience. Circulation 2009;120:S292-8.

8. Song JK, Yim JH, Ahn JM, et al. Outcomes of patients with acute type a aortic intramural hematoma. Circulation 2009;120:2046-52.

9. Shimokawa T, Ozawa N, Takanashi S, et al. Intermediateterm results of surgical treatment of acute intramural hematoma involving the ascending aorta. Ann Thorac Surg 2008;85:982-6.

10. Bossone E, LaBounty TM, Eagle KA. Acute aortic syndromes: diagnosis and management, an update. Eur Heart J 2018;39:739-49d.

11. Lin H, Du Y, Yu C, et al. Single Stage Hybrid Repair for DeBakey Type I Aortic Dissection in High Risk Patients. Eur J Vasc Endovasc Surg 2018;56:363-72.

12. Kaji S, Nishigami K, Akasaka T, et al. Prediction of progression or regression of type A aortic intramural hematoma by computed tomography. Circulation 1999; 100:II281-6.

13. Song JK. Update in acute aortic syndrome: intramural hematoma and incomplete dissection as new disease entities. J Cardiol 2014;64:153-61.

14. Pelzel JM, Braverman AC, Hirsch AT, et al. International heterogeneity in diagnostic frequency and clinical outcomes of ascending aortic intramural hematoma. J Am Soc Echocardiogr 2007;20:1260-8.

15. Watanabe S, Hanyu M, Arai Y, et al. Initial medical treatment for acute type a intramural hematoma and aortic dissection. Ann Thorac Surg 2013;96:2142-6.

16. Sawaki S, Hirate Y, Ashida S, et al. Clinical outcomes of medical treatment of acute type A intramural hematoma. Asian Cardiovasc Thorac Ann 2010;18:354-9.

Cite this article as: Shi Y, Dun Y, Guo H, Liu Y, Zhang B, Qian X, Yu C, Sun X. Clinical features and surgical outcomes of type A aortic intramural hematoma. J Thorac Dis 2020;12(8):3964-3975. doi: 10.21037/jtd-20-748
17. Song JK, Kim HS, Kang DH, et al. Different clinical features of aortic intramural hematoma versus dissection involving the ascending aorta. J Am Coll Cardiol 2001;37:1604-10.

18. Moizumi Y, Komatsu T, Motoyoshi N, et al. Management of patients with intramural hematoma involving the ascending aorta. J Thorac Cardiovasc Surg 2002;124:918-24.

19. von Kodolitsch Y, Csosz SK, Koschyk DH, et al. Intramural hematoma of the aorta: predictors of progression to dissection and rupture. Circulation 2003;107:1158-63.

20. Hata M, Hata H, Sezai A, et al. Optimal treatment strategy for type A acute aortic dissection with intramural hematoma. J Thorac Cardiovasc Surg 2014;147:307-11.

21. Ahn JM, Kim H, Kwon O, et al. Differential clinical features and long-term prognosis of acute aortic syndrome according to disease entity. Eur Heart J 2019;40:2727-36.

22. Estrera A, Miller C 3rd, Lee TY, et al. Acute type A intramural hematoma: analysis of current management strategy. Circulation 2009;120:S287-91.

23. Nienaber CA, von Kodolitsch Y, Petersen B, et al. Intramural hemorrhage of the thoracic aorta. Diagnostic and therapeutic implications. Circulation 1995;92:1465-72.

24. Evangelista A, Dominguez R, Sebastia C, et al. Prognostic value of clinical and morphologic findings in short-term evolution of aortic intramural haematoma. Therapeutic implications. Eur Heart J 2004;25:81-7.

25. Matsushita A, Fukui T, Tabata M, et al. Preoperative characteristics and surgical outcomes of acute intramural hematoma involving the ascending aorta: A propensity score-matched analysis. J Thorac Cardiovasc Surg 2016;151:351-8.

26. Leshnower BG. Type A intramural hematoma: An unstable, unpredictable enigma. J Thorac Cardiovasc Surg 2018;155:910-1.

27. Howard CE, Velasco CE, Roullard CP, et al. Aorto-right atrial fistula after Bentall repair. Proc (Bayl Univ Med Cent) 2017;30:301-2. 